\title{
A Forensic Email Analysis Tool Using Dynamic Visualization
}

Johannes Stadlinger

University of Erlangen-Nuremberg, johannes.stadlinger@fau.de

Andreas Dewald

ERNW Research GmbH

Follow this and additional works at: https://commons.erau.edu/jdfs|

Part of the Computer Engineering Commons, Computer Law Commons, Electrical and Computer Engineering Commons, Forensic Science and Technology Commons, and the Information Security Commons

\section{Recommended Citation}

Stadlinger, Johannes and Dewald, Andreas (2017) "A Forensic Email Analysis Tool Using Dynamic Visualization," Journal of Digital Forensics, Security and Law. Vol. 12 : No. 1 , Article 6.

DOI: https://doi.org/10.15394/jdfsl.2017.1413

Available at: https://commons.erau.edu/jdfsl/vol12/iss1/6

This Article is brought to you for free and open access by the Journals at Scholarly Commons. It has been accepted for inclusion in Journal of Digital Forensics, Security and Law by an authorized administrator of Scholarly Commons. For more information, please contact commons@erau.edu.

(c)ADFSL 


\title{
A FORENSIC EMAIL ANALYSIS TOOL USING DYNAMIC VISUALIZATION
}

\author{
Johannes Stadlinger, Andreas Dewald \\ University of Erlangen, ERNW Research GmbH
}

\begin{abstract}
Communication between people counts to the most important information of today's business. As a result, in case of forensic investigations in big companies, analysis of communication data in general and especially email, as the still most widely used business communication platform with an immense and still growing volume, is a typical task in digital forensics. One of the challenges is to identify the relevant communication partners and structures in the suspects surrounding as quickly as possible in order to react appropriately and identify further targets of evaluation. Due to the amount of emails in typical inboxes, reading through all the mails renders impractical. Therefore, forensic investigators need tools that support them in quickly receiving an impression of a suspect's email communication, identifying the relevant communication partners, and realizing communication patterns in single or even multiple email accounts. We introduce an open source forensic email analysis tool that provides exactly by means of a responsive and interactive graph visualization of email data supported by statistical information.
\end{abstract}

Keywords: Email Forensics, Investigation, Visualization

\section{INTRODUCTION AND MOTIVATION}

Email communication is an indispensable factor in our most widely digitized world. The Radicati Group (Radicati, 2014) publishes research and even predicts still a slightly growth. They also show that email communication is a huge part of our today's business sector as illustrated by Table 1, presenting their results and forecast for business mails sent/received per user/day. But not only daily busi-

\begin{tabular}{rccccc}
\hline & 2014 & 2015 & 2016 & 2017 & 2018 \\
\hline Total & 121 & 126 & 131 & 136 & 140 \\
Received & 85 & 88 & 91 & 95 & 97 \\
Legitimate & 75 & 77 & 79 & 83 & 83 \\
Spam & 10 & 11 & 12 & 12 & 14 \\
Sent & 36 & 38 & 40 & 41 & 43 \\
\hline
\end{tabular}

Table 1. Average number of Business emails sent/received per user and day, 2014 - 2018 (Radicati, 2014)

ness is performed via email, but also criminal activities such as launching threats, blackmailing, organization of terroristic activities, and leakage of sensitive company data. On such frauds, companies often engage external forensic examiners to investigate the email communication of the company to, for example, identify the source of an information leak. Therefore, the examiners are in need of forensic tools which gather the vast amount of email data and offer a platform to investigate the communication in an effective way (Garfinkel, 2010).

\subsection{Contribution}

We present an open source ${ }^{1}$ tool for email forensics that combines different existing visualization approaches in order to provide a dynamic and responsive way to analyze large amounts of email data from multiple mailbox files to identify communication patterns in unknown mailboxes.

\subsection{Outline}

We take a closer look at related work in the next section. After that, we introduce our forensic email analysis tool by presenting the basic structure as well as its features in Section 2. In Section 3, the tool is evaluated by means of functionality and usability. In Section 4, we conclude our work and identify limitations and future work.

\subsection{Related Work}

In the past years, various tools for forensic email investigation - besides known commercial tools - were published. Also, more specific scientific tools with

\footnotetext{
${ }^{1}$ https://www1.cs.fau.de/content/forensic-email-visualization
} 
similar approaches have been developed, which we want to describe here.

In 2009, Meng et. al. (Meng, Wu, Yang, \& Yu, 2009) introduced their framework called Visualized Association inside Emails (VAIE), which helps the investigators to gather evidence in email communication. The tool provides some data mining features, such as a simple key word search, which classifies emails by key words into several categories. For visualization, this tool offers a spring force model and a radial tree model. Also, the way of visualization has much in common, e.g. a spring force model where all mail addresses are represented as nodes with edges that emphasize a communication between them. But the frequency of the communication, e.g. the number of messages which are exchanged between those two parties is not represented in VAIE. Another general problem of this approach is that it has no possibility to handle the more actual $O S T$ files of Microsoft Outlook. Further, it lacks a feature to adjust an appropriate time interval in order to minimize the data instead of always showing all the emails. A weakness of this tool that we address in our implementation is the weighting of the number of emails exchanged between two parties, as well as a missing time frame filter and missing Microsoft Outlook OST file support.

Another framework for email forensics by (Hadjidj et al., 2009) is called Integrated email Forensic Analyse Framework (IEFAF). It is implemented in Java and also provides email visualization. It implements a key word search by using SQL-like queries. Additionally, it provides data mining models to classify messages in different categories and applies authorship analysis (Iqbal, Hadjidj, Fung, \& Debbabi, 2008) on the basis of stylemetric (Abbasi \& Chen, 2008) features in order to identify the most conceivable authors of anonymous messages. A drawback of the tool is that it allows almost no interaction with the resulting graph or charts, as they are are only printed in a static way. It also offers no option to parse existing mailbox files like PST or MBOX.

One of the most known tools is the email Mining Toolkit (EMT) developed by (Li, Hershkop, \& Stolfo, 2004) at the Columbia University. It is an open-source forensic examination tool that computes behavior profiles and models of user email accounts. EMT supports various email storage formats including Microsoft's PST and MBOX, as well as many others (but not the OST format). It offers automatic email classification by applying machine learning techniques and provides a detailed statistical evaluation of the mailbox. The central approach of this system is the underlying data mining and analysis subsystem that provides information such as group behavior, the path a message has taken in a conversation and the user's average response duration. The scope of visualization however differs from our approach: we want to concentrate on the entire communication and offer a simple and unique graphical visualization to get a quick overview of the mail account instead of going into deep message tracking and data mining.

Uforia (Eijkhoudt \& Suerink, 2013) is a simple and extensible framework for analysis and parsing of file meta data. The framework includes possibilities to investigate archives like MBOX and Microsoft Outlook's PST files, too. The front end allows the examiner to filter and search the data for specific senders, recipients, keywords etc. by using the ElasticSearch engine. In contrast to our tool, there is no strong focus on visualizing the email communication to provide the user the opportunity to get a fast and descriptive overview of the communication and then take further actions like adjusting a time interval, and so on.

\section{IMPLEMENTATION}

Our tool is implemented in Python and makes use of JavaScript for the interactive user and exploration interface that is served via a local web server. The tool consists of four modules:

1. Initialization Module

2. Parsing Module

3. Graph Generation Module

4. Visualization and Interaction Module In the following, we provide more detailed information about each module.

\subsection{Initialization Module}

The task of the initialization module is to hand the input data over to the respective parsing module, inserting the parsed data into the database and to run the further modules as follows.

\subsection{Parsing Module}

Our tool supports MBOX, PST and OST files. In order to extract the data from the different data formats, we use our module unboxer.py, which functions as a generic wrapper around different parsing libraries. For the simple MBOX format we use the Python in-built library mailbox. which handles the input as simple dictionaries. Microsoft Outlook's more complex formats - PST and OST - are parsed by the Python-bindings of the libpff (Metz, 2014) project. After normalizing the data set, we finally export all messages to a sqlite database with information like sender, recipient, subject, date, and the 
text content of each email. Now the entire email data corpus in the database can be processed by the graph generation module in the next step.

\subsection{Graph Generation Module}

After parsing and storing the data of the mailbox files to the database, we need to generate the basis of the first visualization: an undirected graph. The nodes of the graph thereby represent email addresses (or contacts respectively, as explained later) and the edges formalize a communication between two nodes. For each node, we additionally serve some meta information like number of conversation partners and amount of sent/received messages, for example. We implement the graph data structure using the Python graph library NetworkX.

To fill the graph, we run SQL queries on the database to select the desired messages and insert them into the graph-object. Thereby, we distinguish if the current sender-recipient-edge is already represented in the graph or we have to add it as a new edge.

After this processing step, we end up with a basic graph that is then exported as a JSON data structure, which then can be handled and dynamically explored within the front end, as described in the next section.

\subsection{Visualization and Interaction Module}

In order to provide a flexible and responsive user interface, our tool provides a web-based user interface utilizing HTML5 and JavaScript with the help of the Flask micro framework. Figure 1 on the following page shows the initial investigation page, which is separated in two areas: the panel on the left gives the examiners meta information about the mailboxes and presents statistic information in different charts and diagrams. On the right, the investigator can access the entire communication data by exploring the graph which the tool built from the data. Both panels deliver a rich set of user interaction possibilities and all displayed information is updated in real time after changes or selections in one of them. We now explain the features of the two panels in detail:

\subsubsection{Meta Panel}

The meta panel offers several different views on the parsed email data. As you can see in Figure 1 on the next page, a bar chart is settled on top and provides a timeline to illustrate the number of emails over time and allows the investigator to select an arbitrary time range as a filter. All the other information is then updated accordingly. To display the amount of written/sent messages by the averaged daytime, we show an additional bar chart. Underneath, there is a top- 15 list of the most active senders of the current selection and a pie chart which represents the data per weekday and a counter of the total emails that fall into the current selection. We use the crossfilter library to calculate and update all the graphs on the fly from the initial data that was provided within the JSON object. With the help of these responsive charts, the investigator is able to filter the entire data by different factors. For example, by clicking on the pie chart's Saturday- and Sunday-slice, all diagrams realign their data, too.

\subsubsection{Exploration Panel}

The exploration panel on the right offers the main functionality of our front end. It is entirely implemented in JavaScript and visualizes the graph by using the d3.js (Bostock, 2011) library. As can be seen from Figure 1 on the following page, the graph consists of blue nodes (the email addresses) and communication between two nodes is illustrated as a link between the nodes. Those links (edges) are of different length and thickness to illustrate the frequency of communication: the shorter and thicker the link (the closer the nodes), the more emails have been exchanged between the communication partners. This way, the main communication partners are grouped to each other and can be easily identified. Besides those two indications, we decided to add toggleable numbers on each edge to show the exact number of emails (see Figure 2 on the next page). An investigator can begin the investigation by exploring the initial graph and check for nodes with many edges or identify the main communication partners by looking for thick edges, for example. Nodes can get "sticky" by just double-clicking on it to make it easier for the user to find it again and rearrange the graph. Further, communication patterns like rings, or outsiders, proxies or others can easily be recognized as shown in Figure 2 on the following page. After clicking on a node, a report of the node's attributes is displayed and the single emails be can read, if necessary.

Initially, we gather all the data from the JSON input that has been generated by the graph generation module to build the visual graph. After creating a basic graph-object and adjusting the visual properties and distances accordingly, we register functions to resolve user events like click or mouseover to the node objects. Those provide the investigator the ability to interactively analyze the entire communication by reordering the graph, pulling out or grouping nodes, or clicking on them to show more 


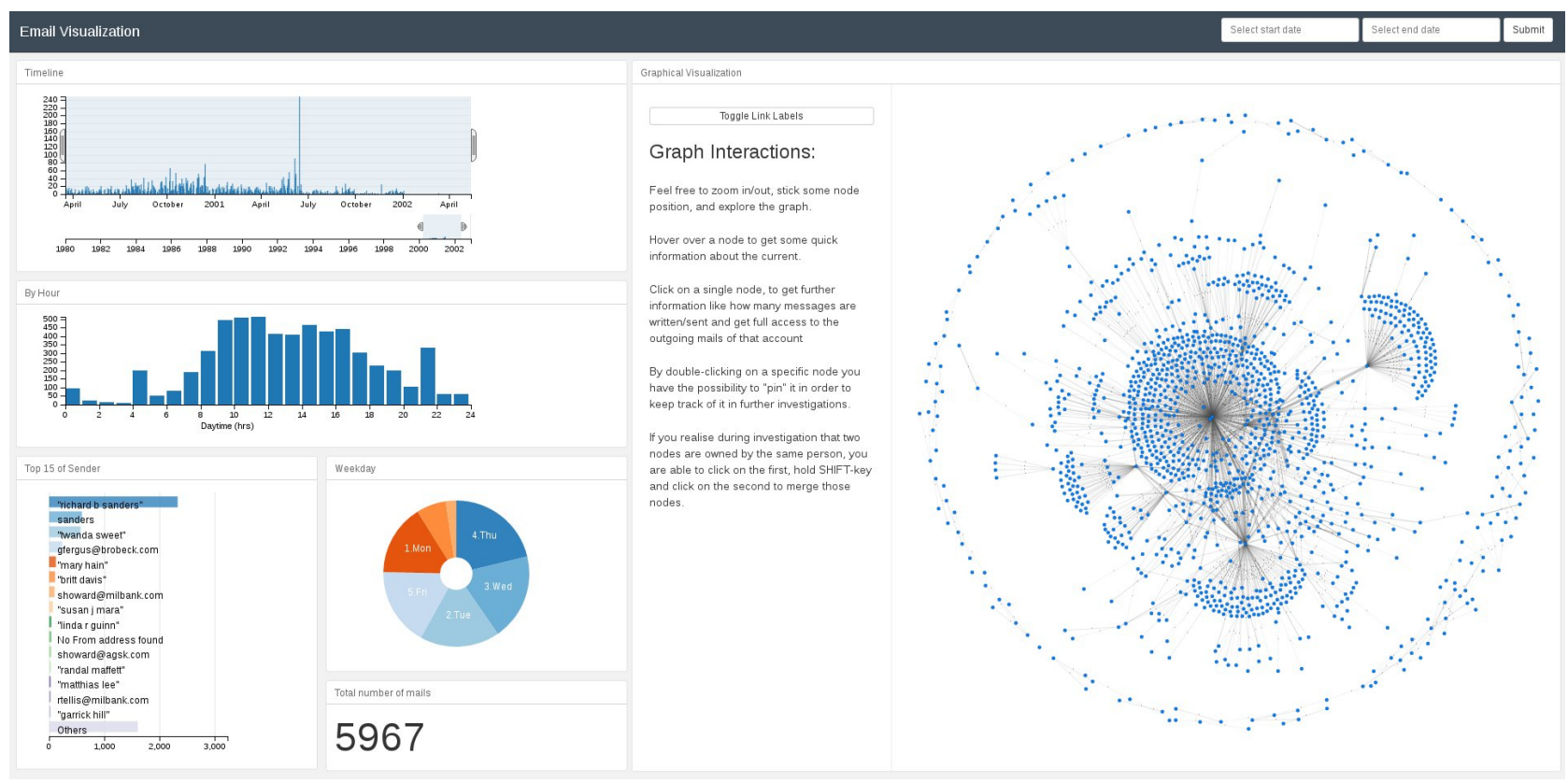

Figure 1. Investigation page with meta panel on the left and graph visualization on the right.

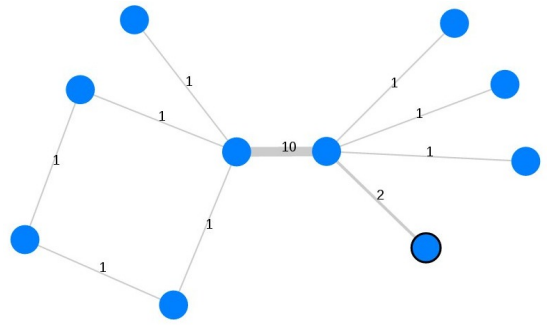

Figure 2. Simple example of a communication ring pattern in email data. A thicker edge highlights an increased communication frequency.

information - down to every single email. Additional to the side panel, our tool provides some basic node information: when the investigator hovers over a specific node, a pop-up window appears and shows the email address, number of conversation partners, and the number of sent and received emails.

As there might be multiple email addresses belonging to the same person within an investigation data set, the investigator can select two nodes by holding down the shift key while clicking to group them together. All attributes are updated and the resulting combined node is highlighted in red.

\section{EVALUATION}

In order to evaluate the practicability of our tool, we used parts of the ENRON email collection (Klimt \& Yang, 2004). From that collection we randomly picked ten PST files with different message counts from 500 up to 10,000 emails as shown in Table 2.

All tests are executed on our test system with an Intel Core i5-4670 quad-core processor with a clock rate of $3,400 \mathrm{MHz}$ and 16 gigabytes of main memory. The operating system is a Debian 8 "Jessie" 64-bit.

\subsection{Performance}

We first measured runtime performance of our parsing modules for PST/OST files of different sized mailboxes. The results are summarized in Table 2. For parsing PST/OST files, the parsing of around 5,000 messages took 1.23 seconds and 10, 000 needed around 2.89 seconds.

\begin{tabular}{rcl}
\hline \#Messages & Time & ENRON-file \\
\hline 483 & $0.159 \mathrm{~s}$ & bill_rapp_000_1_1.pst \\
1,013 & $0.301 \mathrm{~s}$ & andrea_ring_000_1_1.pst \\
1,538 & $0.355 \mathrm{~s}$ & benjamin_rogers_001_1_1.pst \\
2,660 & $0.462 \mathrm{~s}$ & lindy_donoho_000_1_1_1.pst \\
4,099 & $1.146 \mathrm{~s}$ & don_baughman_000_1_1.pst \\
4,942 & $1.226 \mathrm{~s}$ & kenneth_lay_000_1_1_1_1.pst \\
6,091 & $1.492 \mathrm{~s}$ & Richard_Sanders_001_1_1_1.pst \\
7,550 & $1.796 \mathrm{~s}$ & andy_zipper_000_1_1.pst \\
8,500 & $2.384 \mathrm{~s}$ & gerald_nemec_001_1_1.pst \\
10,000 & $2.897 \mathrm{~s}$ & richard_sanders_001_1_2.pst \\
\hline
\end{tabular}

Table 2. Parsing performance of PST/OST files.

For MBOX files, the runtime increases approximately linearly with the number of messages. For example, parsing 5,000 messages took 7.38 seconds and 10,000 needed 14.88 seconds. This shows that 
the parsing library used for PST and OST files is much faster than the MBOX parser. This could be improved by implementing an own, optimized MBOX parsing library, but the runtime performance of both formats should still be good enough to allow the use of our framework in real world cases.

\subsection{Correctness and Completeness}

In this section, we try to argue on the correctness and completeness of the generated graphs, by proving some specific scenarios. For each scenario, we previously specified the expected correct behavior and then checked, whether the output and behavior of our tool corresponds to these.

\section{Scenario 1}

Issue Are all messages represented in the graph with their right attributes (like number of sent/received emails)?

Input The mailbox includes one mail account, which has written four and received eight messages. All written/received messages have a single unique target.

Expected behavior A forced directed graph with one centered node with edges to twelve other nodes, which are representing the source and target of the involved messages. The attributes of the center node should contain twelve conversation partners, as well as four written and four received messages. In the list there should be four clickable messages with their content. All nodes should have the same distance to the center node and edges should all have the same thickness.

Result Test accomplished (see resulting graph in Figure 3).

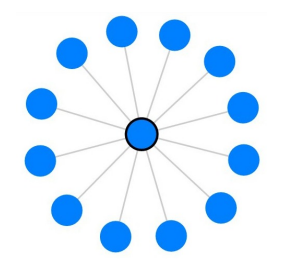

Figure 3. Resulting graph of Scenario 1.

\section{Scenario 2}

Issue That test evaluates if the attributes of the inserted nodes are treated correctly, i.e. does the graph recognize all messages and differs between sent and received.

Input One mail account, which has written four mail to two different recipients (i.e. two mails to $A$ and two mails to $B$ ) and received four messages.
One received from $A$ and the others from three not previously involved accounts.

Expected behavior A graph with six nodes. The mail account acts as the center from which edges spread out to the other five nodes. The registered attributes of the center node should be five conversation partners, four written, four received messages as well as a list of the sent four mails. Node $A$ has sent one mail and received two. Node $B$ has received two and sent zero. The others only sent one message and should have a higher distance and thinner edge to the center node.

Result Test accomplished (see Figure 4).

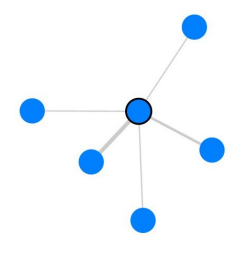

Figure 4. Resulting graph of Scen02: One center which has four edges to its contacts. One link is thicker than the others.

\section{Scenario 3}

Issue This test proves the visualization in order to represent a more complex communication between multiple mail accounts.

Input Three mailbox files: account $A, B$ and $C$. User $A$ has written one to $B$ and one message to $C$ as well as two mails to an external account $D$. Account $B$ has written only one message to $D$. User $C$ has written four mail to $D$ and one message each to three other external persons.

Expected behavior A cohesive forced graph appears with seven individual nodes. One of the nodes represents $A$ from which three links are spread out to $B, C$, and $D$. The edge between $A$ and $D$ is shorter and thicker. Node $B$ has two links, the first to $A$ and the seconds to $D$. Followed by node $C$ which has six edges where one links to $D$, one to $A$ and four to other nodes.

Result Test accomplished (see Figure 5).

This just shows a selection of some of the performed tests, the rest of our test series has to be omitted here due to the page limitation.

\section{CONCLUSION}

In this section, we want to summarize our paper and discuss limitations, as well as future work. 


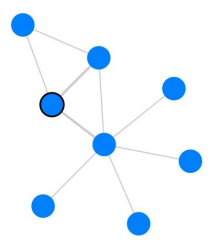

Figure 5. Graphical representation of a more complex communication.

\subsection{Summary}

We implemented a tool for forensic email analysis using dynamic visualization approaches. Our tool is able to handle multiple mailboxes of three of the most common formats to cover as many cases as possible. Mailboxes of different formats can be used at the same time. The centerpiece of our application is the graphical visualization of the email communication. It allows the investigators to identify suspicious patterns in unknown email communication and quickly see who are the main contacts. Our tool allows users to select a specific time range, group and move nodes manually and updates all information and charts in real time.

\subsection{Limitations}

The current state of the developed tool is fully functional as described. However, we see the following directions for improvements:

First of all, we might improve parsing speed performance especially for MBOX files by optimizing the input parsers. Further, parsers for other formats, like Microsoft's Electronic Mail (EML), whole Maildirs, Message Handling (MH), or others could be added.

We further would like to verify that all kinds of malformed messages that can occur in reality are handled correctly.

\subsection{Future Work}

Besides the limitations we considered above, we plan to enhance the UI to cover the entire process of creating, saving and loading cases and importing email data to it. We further want to implement a fulltext keyword search for the email data, and make attachments searchable and viewable, too.

We also plan to implement the possibility to directly generate forensics reports, which describe what has been done during the investigation and export results and bookmarked emails or similar.

\subsection{Conclusion}

We conclude that we have developed a forensic email analyzing tool which helps the investigator to iden- tify patterns and clues in a suspected communication. It offers the results in an innovative, dynamical way of visualization using forced directed graphs and responsive charts. Although there are some limitations and ideas for future work left as discussed before, we provided a practical open source tool that might help investigators in today's email forensics cases and provides a valuable lineup for existing forensic toolchains.

\section{REFERENCES}

Abbasi, A., \& Chen, H. (2008). Writeprints: A stylometric approach to identity-level identification and similarity detection in cyberspace. ACM Transactions on Information Systems (TOIS), 26(2), 7.

Bostock, M. (2011). D3-Data-Driven-Documents. Retrieved 2016-05-18, from http://d3js.org/

Eijkhoudt, A., \& Suerink, T. (2013). Uforia: Universal forensic indexer and analyzer. Journal of Computer Virology and Hacking Techniques, 9(2), 59-63.

Garfinkel, S. L. (2010). Digital forensics research: The next 10 years. digital investigation, 7 , S64-S73.

Hadjidj, R., Debbabi, M., Lounis, H., Iqbal, F., Szporer, A., \& Benredjem, D. (2009). Towards an integrated e-mail forensic analysis framework. digital investigation, 5(3), 124-137.

Iqbal, F., Hadjidj, R., Fung, B. C., \& Debbabi, M. (2008). A novel approach of mining write-prints for authorship attribution in e-mail forensics. digital investigation, 5, S42-S51.

Klimt, B., \& Yang, Y. (2004). Introducing the enron corpus. In Ceas.

Li, W.-J., Hershkop, S., \& Stolfo, S. J. (2004). Email archive analysis through graphical visualization. In Proceedings of the $2004 \mathrm{acm}$ workshop on visualization and data mining for computer security (pp. 128-132).

Meng, F., Wu, S., Yang, J., \& Yu, G. (2009). Research of an e-mail forensic and analysis system based on visualization. In Computational intelligence and industrial applications, 2009. paciia 2009. asia-pacific conference on (Vol. 1, pp. 281-284).

Metz, J. (2014). libpff library. Retrieved 2016-05-18, from https://github.com/libyal/libpff 
Radicati, S. (2014). Email statistics report, 2014-2018. the radicati group. Inc., London. 
